\title{
Evolution of the free volume between rough surfaces in contact
}

\author{
M. Paggi ${ }^{\mathrm{a}, \mathrm{b}, *}$, Q.-C. $\mathrm{He}^{\mathrm{a}}$ \\ ${ }^{a}$ Université Paris Est, Laboratoire Modélisation et Simulation Multi Echelle, MSME UMR \\ 8208 CNRS, 5 Bd Descartes, 77454 Marne-la-Vallée Cedex 2, France \\ ${ }^{b}$ IMT Institute for Advanced Studies Lucca, Piazza San Francesco 19, 55100 Lucca, Italy
}

\begin{abstract}
The free volume comprised between rough surfaces in contact governs the fluid/gas transport properties across networks of cracks and the leakage/percolation phenomena in seals. In this study, a fundamental insight into the evolution of the free volume depending on the mean plane separation, on the real contact area and on the applied pressure is gained in reference to fractal surfaces whose contact response is solved using the boundary element method. Particular attention is paid to the effect of the surface fractal dimension and of the surface resolution on the predicted results. The free volume domains corresponding to different threshold levels are found to display fractal spatial distributions whose bounds to their fractal dimensions are theoretically derived. A synthetic formula based on the probability distribution function of the free volumes is proposed to synthetically interpret the numerically observed trends.
\end{abstract}

Notice: this is the authors version of a work that was accepted for publication in Wear. Changes resulting from the publishing process, such as editing, structural formatting, and other quality control mechanisms may not be reflected in this document. A definitive version was published in Wear, Vol. 336, 86-95, DOI:10.1016/j.wear.2015.04.021

Keywords: Rough surfaces; Contact mechanics; Boundary element method; Evolution of the free volume.

\section{Introduction}

Contact mechanics between rough surfaces is a topic of paramount importance in engineering and physics, since surface phenomena in nature and technology strongly depend on the topological properties of interfaces. Real surfaces

\footnotetext{
${ }^{*}$ Corresponding author. Tel: +39-0583-4326-604, Fax: +39-0583-4326-565

Email addresses: marco.paggi@imtlucca.it (M. Paggi), qi-chang.he@univ-paris-est.fr (Q.-C. He)
} 
are never ideally flat and roughness is present at different scales, from the specimen size down to the interatomic distance. Hence, when two bodies are pressed against each other, contact takes place at the asperities (the 3D maxima of the surfaces) and the real contact area is a fraction of the nominal one.

In the context of rough surfaces, the scientific community has paid particular attention to the relation between the real contact area and the applied pressure [1, 2, 3, 4, 5, 6, 7, the contact stiffness [8, 9, 10, 11, 12, 13, which is proportional to the electric and thermal contact conductances [14, 15, 16, 17, frictional phenomena [18, 19, adhesion [20, 21], and hydrophobic properties of surfaces [22, 23. Recent studies on rough surfaces have also elasto-plastic contact [24, 25], adhesive contact [26], and lubrication [27.

Another important topic regards the transport properties of rough surfaces in contact. Below the full contact limit, a free volume between the contacting bodies is always present due to roughness. Such a free volume constitutes a fractal network whose properties are important for flow and transport of hydrothermal fluids, water, and contaminants in groundwater systems, but also of oil and gas in petroleum reservoirs [28. For instance, the transport properties of proppant through fracture networks are relevant for hydraulic fracturing [29]. At a much smaller scale, welded surfaces in micro-electro-mechanical systems (MEMS) may present a free volume forming channels and capillaries of random distribution. Such channels are critical for gas leakage that may penetrate the soldered joint and affect the reliability of the system [30]. These problems are also relevant in materials for energy applications, such as in solid oxide fuel cells 31 and in photovoltaic modules where humidity can diffuse along the interface between the textured surface of solar cells and the encapsulating polymer, promoting a chemical degradation of electric contacts. The topological features of roughness in seal contacts are also very important for the onset of wear, see 32 .

Attempts to predict the transport properties across these finite thickness interface regions composed of voids and contact areas are relatively recent and rely on the theory of fractal porous media [33, 34]. Based on this modelling assumption, simplified theories are put forward where the free volumes are treated as pores of spherical shape with diameter obeying a power-law distribution. Pioneering analytical models have been proposed in [35, 36, 37] by examining the evolution of the contact area depending on the surface resolution. For a flat surface, the full contact regime takes place and no percolation channels are present. By refining the surface resolution, roughness comes into play and the real contact area becomes a fraction of the nominal one. For a given critical resolution, a first percolating channel will be originated. Further surface refinements will lead to other percolation channels that may contribute to the global leakage rate. Such contributions have been neglected in [35, 36, 37]. Due to such simplifying assumption, predictions were found in good agreement with experimental results only in the low pressure regime.

A rigorous computational approach to predict the contact area and the transmissivity and diffusivity of the network of the created free channels was recently proposed in 38, where the problem was tackled from the numerical point of view by using the boundary element method (BEM). However, the analysis was 
restricted to two specific surface topologies created by lapping or sand blasting treatments and general trends were not discussed.

In the present study we propose an extensive numerical investigation of the evolution of the free volume between fractal rough surfaces in contact with an elastic half plane as a function of the main contact variables, i.e., the mean plane separation, the contact force and the real contact area. A computational approach based on BEM, analogous to that described in [38], is used. A deep analysis of the morphological properties of the free volume domains is performed, without making simplifying assumptions a priori on their shape and distribution, as in previous models based on the percolation theory. Moreover, all the channels are considered without any approximation apart from that arising from the spatial discretization intrinsic in the method. The obtained numerical trends and their interpretation are expected to provide useful hints for the development of further semi-analytical models taking into account the observed scaling laws, or to refine the existing ones.

The article is structured as follows. In Section 2, the numerical method used to generate the rough surfaces is outlined and the fundamental equations of the boundary element method used to solve the contact problem are described. In Section 3, numerical results are presented and focus on the scaling of the free volume and on the multi-scale characterization of its network pattern. Further theoretical considerations on the statistical distribution of the free volumes are provided in Section 4, along with a synthetic formula for the computation of the free volume and for a deeper understanding of the observed numerical trends. Conclusions and outlook on the relevance of the proposed methodology for the study of wear in seal applications complete the study.

\section{Numerical method}

Rough surfaces with fractal properties are numerically generated according to the random midpoint displacement (RMD) algorithm [39]. This method allows generating rough surfaces with a power spectral density function of powerlaw type, characterized by a given fractal dimension $D(2<D<3)$. Applications of the method to contact mechanics can be found in [3, 6, 40]. Square surfaces with different resolutions can be generated by successively refining an initial mesh by a successive addition of a series of intermediate heights. In the algorithm, the number of successive refinements is defined by the parameter $m$, which is related to the number of heights per side of the squared generated grid, $2^{m}+1$. Given $L$ the lateral size of the surface, the grid spacing is $\delta=L / 2^{m}$ and the resolution can be defined as $s=1 / \delta$. The method generates surfaces with higher $m$ that are finer representations of the coarser ones, i.e., the height field of a surface with $m=i, i \in \mathbb{N}$, contains the height field of the coarser realizations with $m<i$.

A sketch showing how the RMD algorithm operates is provided in Fig 1 Starting with $m=1$, the elevation of the four corner nodes of the grid, nodes $o, p, j, s$ in Fig,1, are set equal to zero. Afterwards, the elevation of the central point of the grid, $l$, is determined by the average value of the elevations of the 

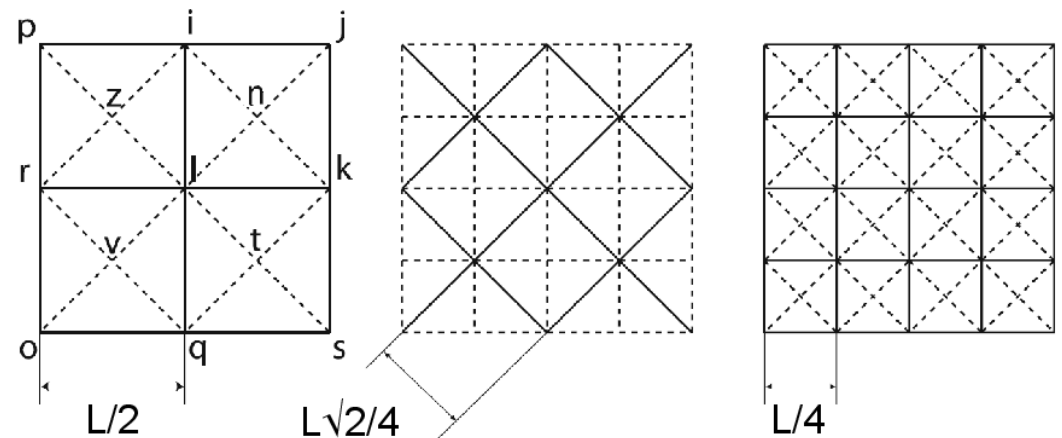

Figure 1: Recursive steps for the generation of rough surfaces using the RMD algorithm.

corner nodes, plus a random number extracted from a Gaussian distribution with zero mean and variance $\sigma_{1}^{2}=\sigma_{0}^{2} / 2^{(3-D) / 2}$, where $\sigma_{0}^{2}$ is a free parameter set equal to $1 / \sqrt{0.09}$. The elevations of the nodes $i, k, q, r$ are then assigned by averaging over three elevations, those of the two corner nodes and that of the central node, plus a random number extracted from a Gaussian distribution with zero mean and reduced variance $\sigma_{2}^{2}=\sigma_{1}^{2} / 2^{(3-D) / 2}$. This procedure is further iterated at the next refinement, $m=2$. This version differs from the original RMD algorithm detailed in 39] by the fact that the elevations of the four initial corner nodes are set equal to zero rather than randomly assigned. The reason is to avoid to create topologies dominated by these initial values, which might constitute a bias especially at low resolution.

The most accurate solution of the contact problem between the generated rough surfaces and a smooth plane by keeping as minimum as possible the simplifying assumptions on the surface geometry can be achieved by using the boundary element method for contact mechanics [41]. By imposing a far-field closing displacement $\Delta$ to the bodies in contact, the displacement at each point of the contact area is related to the contact pressures as follows [42:

$$
u(\mathbf{x})=\int_{S} H(\mathbf{x}, \mathbf{y}) p(\mathbf{y}) \mathrm{d} S,
$$

where $u(\mathbf{x})$ is the displacement at the surface point defined by the position vector $\mathbf{x}, H(\mathbf{x}, \mathbf{y})$ is the displacement at $\mathbf{x}$ due to a unit pressure acting at $\mathbf{y}$, and $S$ is the apparent contact area. Assuming linear elastic isotropic materials, the influence coefficients are given by [41, 42]:

$$
H(\mathbf{x}, \mathbf{y})=\frac{1-\nu^{2}}{\pi E} \frac{1}{\|\mathbf{x}-\mathbf{y}\|},
$$

where $E$ and $\nu$ denote, respectively, the composite Young's modulus and Poisson's ratio of the materials of the bodies in contact. Upon discretization of the surface as a grid where each nodal height defined by the indices $(i, j)$ is modelled as a square punch with an elevation $\xi_{i, j}$ above a reference plane coincident 
with the level of the smallest surface height, the contact problem requires the simultaneous solution of the following set of equations and the satisfaction of a set of inequalities:

$$
\begin{aligned}
& u_{i, j}=\sum_{k=1}^{N_{c}} \sum_{l=1}^{N_{c}} H_{i-k, j-l} p_{k, l}, \quad 1 \leq i \leq N_{c}, \quad 1 \leq j \leq N_{c} \\
& u_{i, j}=\bar{u}_{i, j}=\Delta-\xi_{i, j}, \quad \forall(i, j) \in \mathcal{I} \\
& p_{i, j}>0, \quad \forall(i, j) \in \mathcal{I} \\
& u_{i, j}>\bar{u}_{i, j}, \quad \forall(i, j) \in \overline{\mathcal{I}} \\
& p_{i, j}=0, \quad \forall(i, j) \in \overline{\mathcal{I}}
\end{aligned}
$$

where $\mathcal{I}$ is the domain of boundary elements in contact, $\overline{\mathcal{I}}$ is the domain of boundary elements not in contact, and $\Delta$ is the imposed far-field displacement. An initial (trial) contact domain is chosen as the set $\mathcal{I}_{0}$ of boundary elements that compenetrate in the half-plane in case of a rigid body motion, i.e., by neglecting the deformation induced by elastic interactions. The number of boundary elements belonging to this set is $N_{c}$. The optimal contact domain is found iteratively by a suitable elimination of the points bearing tensile (negative) forces [41, 43. During contact, the evolution of the gaps between the surface grid points and the half-plane as a result of the elastic interactions is traced. From the computed normal displacements, the free volume of each boundary element is $v_{i, j}=\left(u_{i, j}-\bar{u}_{i, j}\right) \delta^{2}$. All the points belonging to the contact domain, $\mathcal{I}$, have by definition $u_{i, j}=\bar{u}_{i, j}$ and therefore $v_{i, j}=0$. The total free volume $V$ is finally evaluated by summing up the individual boundary element contributions.

The main steps of the contact algorithm are summarized in Algorithm 1, where $\mathbf{H}$ denotes the matrix collecting the influence coefficients, the vector $\overline{\mathbf{u}}$ collects the imposed displacements, and the vector $\mathbf{p}$ collects the contact forces. The boundary elements subject to tensile forces are eliminated from the contact domain at the step (2.2.1). After convergence, the optimal solution is stored in the vector $\mathbf{p}_{\text {opt }}$ in the step (3). The set of boundary elements not in contact, $\overline{\mathcal{I}}$, is updated in the step (4). The displacements of the whole surface are computed in the step (5) and they are equal to $\overline{\mathbf{u}}_{\mathcal{I}}$ for the elements in contact, whereas they are given by the elastic equations for the elements not in contact, using the corresponding flexibility matrix $\mathbf{H}_{\overline{\mathcal{I}}, \mathcal{I}}$.

The output is given by the following dimensionless quantities: the real contact area fraction, $A^{*}:=A / A_{n}$, where $A_{n}=L^{2}$; the dimensionless pressure, $p^{*}:=p L /(E \sigma)$, where $E$ is the composite Young's modulus of the contacting bodies and $\sigma$ is the r.m.s. of the distribution of surface heights, also called $S_{q}$ in [44, 45]; the dimensionless free volume, $V^{*}:=V /\left(L^{2} \sigma\right)$.

The above dimensionless formulation has been chosen in order to have results invariant with respect to a transformation of the in plane coordinates of the type $x \rightarrow \lambda x, y \rightarrow \lambda y(\lambda \in \mathbb{R})$. Such a linear mapping leads to $L \rightarrow \lambda L, p \rightarrow p / \lambda$, and $V \rightarrow \lambda^{2} V$. Hence, the dimensionless pressure $p^{*}$ and the dimensionless volume $V^{*}$ defined as before are not affected by this type of scaling. However, it has to 
be pointed out that this is true if and only if asperities deform linear elastically. Hence, a lower bound to the surface dimension $L$ that can be explored for a given number of heights per side without the occurrence of plastic deformation does exist. Such a critical lateral size, $L_{c}$, can be identified for a given material by setting the plasticity index equal to unity. Using for instance the expression proposed by Greenwood and Williamson [1], $\psi=\sqrt{\sigma / \rho} E / H$, where $H$ is the material hardness and $\rho$ is the average radius of curvature of the asperities (which is the only parameter entering $\psi$ affected by the linear mapping), we obtain the critical radius of curvature $\rho_{c}=\sigma(E / H)^{2}$ below which asperities are expected to deform plastically. Therefore, any scaling of the type $L \rightarrow \lambda L$ with $\lambda<1$ and leading to $\rho \rightarrow \lambda \rho$ leads to the same $p^{*}$ and $V^{*}$ computed for $\lambda=1$ provided that $\rho>\rho_{c}$. For $\rho \leq \rho_{c}$, the present computational model should be extended by taking into account plastic deformations.

$\overline{\text { Algorithm } 1 \text { Contact algorithm for a given imposed far-field displacement } \Delta}$

Input: Matrix $\mathbf{H}$, vector $\overline{\mathbf{u}}$, initial guess $\mathbf{p}$, initial active set $\mathcal{I}_{0}$, maximum number $K_{\max }$ of iterations, tolerance $\epsilon$.

1. $k \leftarrow 0, \mathcal{I} \leftarrow \mathcal{I}_{0}$;

2. while $\left(k \leq K_{\max }\right.$ and $\left.\min (\mathbf{p})<-\epsilon\right)$ or $k=0$ do:

(2.1) Solve the unconstrained system of equations $\mathbf{H p}=\overline{\mathbf{u}}$ for $\mathbf{p}$ using the Gauss-Seidel algorithm

(2.2) for $(i, j) \in \mathcal{I}$ do:

$(2.2 .1)$ if $p_{i, j}<-\epsilon$ then $p_{i, j} \leftarrow 0 ; \mathcal{I} \leftarrow \mathcal{I} \backslash\{(i, j)\}$

3. $\mathbf{p}_{\text {opt }} \leftarrow \mathbf{p}$

4. $\overline{\mathcal{I}} \leftarrow\left\{\left(1, \ldots, i, \ldots, N_{c}\right) \times\left(1, \ldots, j, \ldots, N_{c}\right)\right\} \backslash \mathcal{I}$

5. $\mathbf{u}_{\mathcal{I}}=\overline{\mathbf{u}}_{\mathcal{I}}, \mathbf{u}_{\overline{\mathcal{I}}} \leftarrow \mathbf{H}_{\overline{\mathcal{I}}, \mathcal{I}} \mathbf{p}_{\mathcal{I}}$

6. end.

Output: dimensionless nominal pressure $p^{*}$, dimensionless contact area $A^{*}$, and dimensionless free volume $V^{*}$.

\section{Numerical results}

\subsection{Scaling of the real contact area and of the free volume}

In the contact between bodies with fractal boundaries and multi-scale roughness, previous research 2, 3, 4, 40 has highlighted the important role of the fractal dimension and of the surface resolution (lower cut-off length) on the relation between the real contact area and the contact pressure. Moreover, the contact domain was topologically characterized in [3] and it was found to be of lacunar type, with fractal properties dependent on those of the parent undeformed fractal surface and on the applied pressure. 


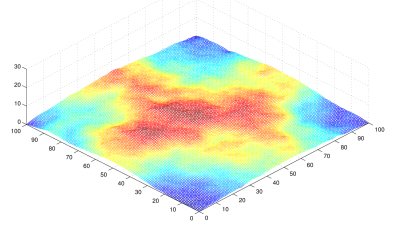

(a) $D=2.1$

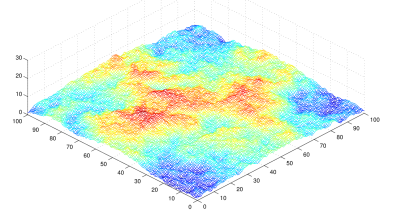

(b) $D=2.5$

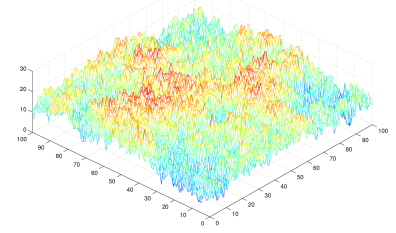

(c) $D=2.9$

Figure 2: the effect of the fractal dimension $\mathrm{D}$ on the numerically generated surfaces with $m=7$ : the increase of roughness with augmenting $D$.

To investigate how the free volume between rough surfaces in contact scales with the real contact area and with the contact pressure, we herein perform a series of contact simulations by using the numerical method described in the previous section. All the numerical tests are performed under displacement control. A displacement $\Delta$ is imposed in the normal direction to the flat contacting plane. The maximum value of $\Delta$ is equal to the difference between the elevation of the highest asperity and the elevation of the mean plane of the asperity heights computed in the undeformed configuration. This total displacement is subdivided in 60 steps.

For each imposed normal displacement, the grid points in contact are determined and the real contact area $A$ and the contact pressure $p$ computed by summing up the contributions of the individual square punches. The volume $V$ comprised between the flat plane and the rough surface is finally determined from the computed displacement field and the original surface geometry according to the method described in Section 2. Numerical results are interpreted via the following dimensionless quantities: the real contact area fraction, $A^{*}=A / A_{n}$; the dimensionless pressure, $p^{*}=p L /(E \sigma)$; the dimensionless free volume, $V^{*}=V /\left(L^{2} \sigma\right)$.

We investigate both the effect of the fractal dimension $D$, see an example in Fig 2, and the effect of the surface resolution by varying the generation parameter $m$, see Fig 3 .

In agreement with previous findings [3], the computed $A^{*}$ vs. $p^{*}$ curves depend on the surface fractal dimension $D$, see Fig $4(\mathrm{a})$ obtained for surfaces with $L=100$ and a resolution determined by $m=8$, viz., 257 heights per side. Monte Carlo simulations have been performed by considering 10 randomly generated surfaces for each set of surface parameters. The larger the fractal dimension $D$, the more spiky the surface with a consequently reduced real contact area for the same given pressure. The relation between $A^{*}$ and $p^{*}$ is also resolutiondependent due to the lacunarity of the contact domain, see Fig 4(b) for surfaces with $D=2.3, L=100$ and different $m$. In this case we just show the results for a single surface, since the trend is the same regardless of the statistical variability in the surface generation. This property was pointed out in theory [2, 4] and in numerical simulations [3] and it implies a vanishing real contact area in the 


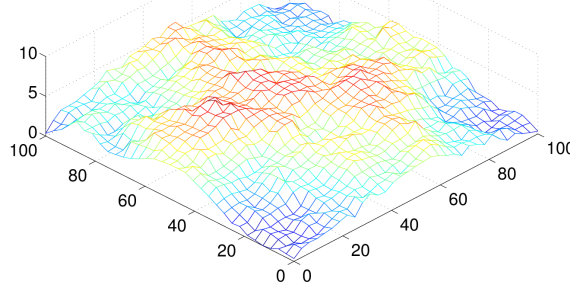

(a) $m=5$

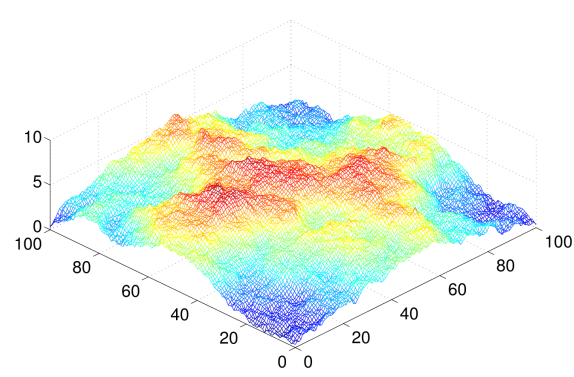

(c) $m=7$

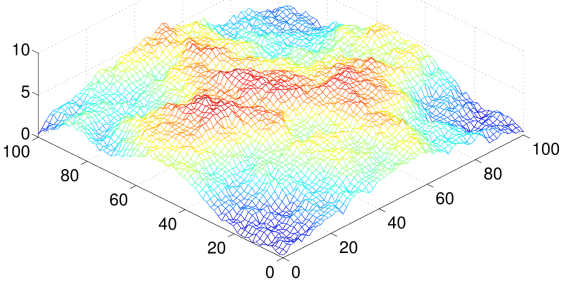

(b) $m=6$

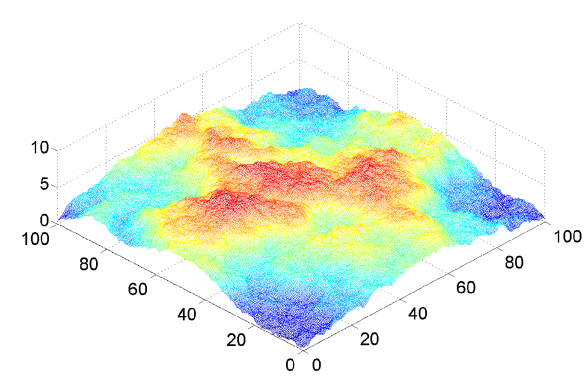

(d) $m=8$

Figure 3: rough surfaces with different values of the parameter $m$ 


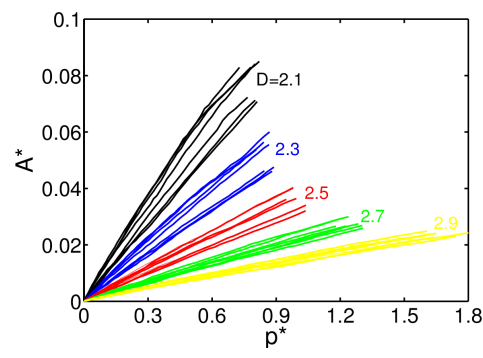

(a) The effect of $D$

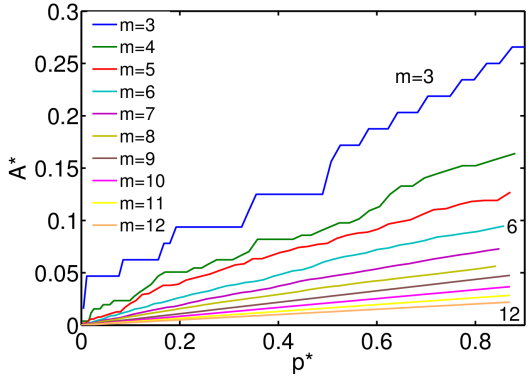

(b) The effect of $m$

Figure 4: Real contact area fraction $A^{*}$ vs. dimensionless pressure $p^{*}$ for numerically generated fractal surfaces. (a) The effect of the fractal dimension $D(10$ surfaces per set, $m=8)$; (b) the effect of the resolution parameter $m$ (surfaces with $D=2.3$ ).

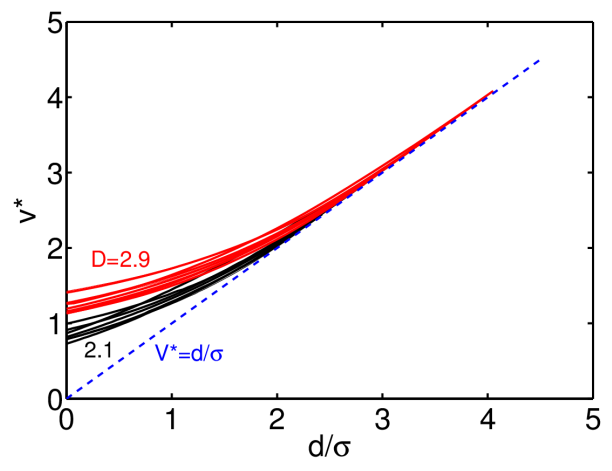

Figure 5: Dependence of $V^{*}$ on the mean plane separation $d / \sigma$. Note the deviation from linearity for small values of $d / \sigma$.

theoretical limit of an infinite resolution $(\delta \rightarrow 0$, or $s \rightarrow \infty)$. Considering a linear approximation for the $A^{*}$ vs. $p^{*}$ relation, we obtain a power-law relation of the type $A^{*} / p^{*} \sim \delta^{0.37}$, which is in fair good agreement with the predictions by Persson theory [4] suggesting $A^{*} / p^{*} \sim \delta^{D-2}$, i.e. $A^{*} / p^{*} \sim \delta^{0.3}$ for the present set of surfaces with $D=2.3$.

As far as the free volume between rough surfaces is concerned, one might argue that this quantity should be somehow proportional to the dimensionless mean plane separation, $d / \sigma$, where $d$ is the separation between the half plane of the rough surface and the elastic half space, and $\sigma$ is the r.m.s. roughness. However, from Fig 5 where the contact predictions for two sets of surfaces with $D=2.1$ (very smooth) and $D=2.9$ (very rough) are depicted, we achieve the important result that the relation $V^{*}=d / \sigma$ (depicted with a dashed blue line) holds only for very large separations $(d / \sigma \gtrsim 3)$. For $(d / \sigma \lesssim 3)$, these two quantities cannot be confused any longer and the free volume starts depending on the fractal dimension $D$ as well. 


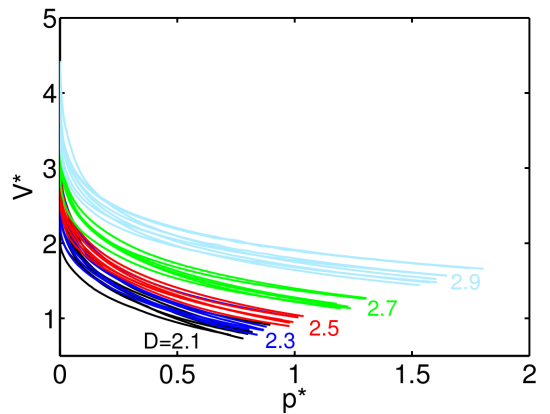

(a) $V^{*}$ vs. $p^{*}$

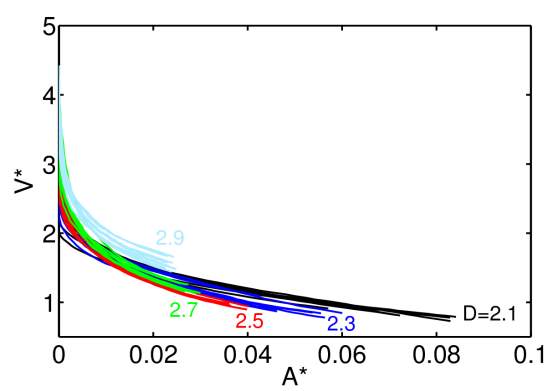

(b) $V^{*}$ vs. $A^{*}$

Figure 6: Dimensionless volume $V^{*}$ vs. dimensionless nominal contact pressure $p^{*}$ and real contact area fraction $A^{*}$, for various fractal dimensions $D$ (10 surfaces per set, $\left.m=8\right)$.

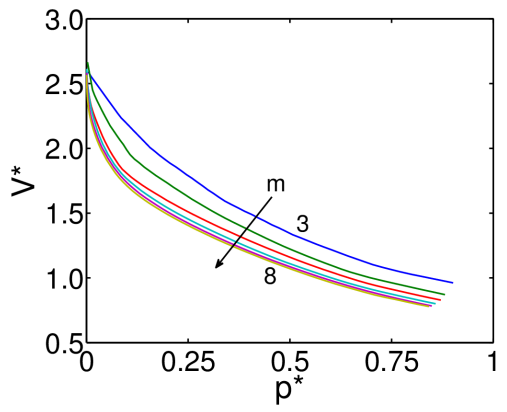

(a) $V^{*}$ vs. $p^{*}$

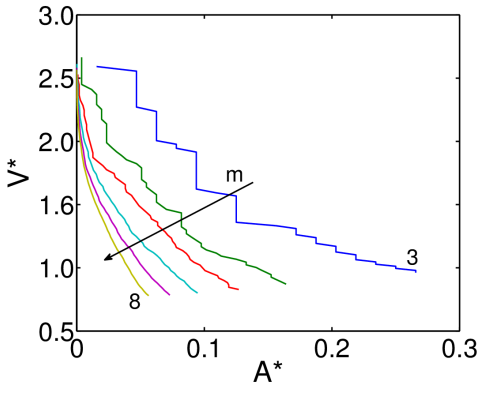

(b) $V^{*}$ vs. $A^{*}$

Figure 7: Dimensionless volume $V^{*}$ vs. dimensionless nominal contact pressure $p^{*}$ and real contact area fraction $A^{*}$, for various values of the surface resolution parameter $m$ ad $D=2.3$.

Investigating the relation between $V^{*}$ and the other contact quantities, namely $A^{*}$ and $p^{*}$, by varying $D$, we obtain the diagrams shown in Fig 6 , A decay of the free volume by increasing the dimensionless contact pressure or the dimensionless real contact area is observed. The relation between $V^{*}$ and $p^{*}$ is significantly affected by $D$ (Fig $6(\mathrm{a})$. . On the other hand, as a notable result, the relation between $V^{*}$ and $A^{*}$ seems to be almost independent of the fractal dimension, since all the curves lie in a relatively narrow band $($ Fig $6(\mathrm{~b}))$.

To examine the role played by the surface resolution, we now consider a single surface with $D=2.3$ and we change the resolution parameter $m$. The trends shown in Fig 7 pinpoint a convergence of the relation $V^{*}$ vs. $p^{*}$ by increasing $m$. On the other hand, the relation $V^{*}$ vs. $A^{*}$ is strongly resolution dependent.

\subsection{Fractal properties of the free volume domains}

From the results of numerical simulations it is possible to visualize the deformed configuration of the rough surface in contact with the half-plane for each imposed displacement, see the undeformed shape of a surface with $D=2.3$, 


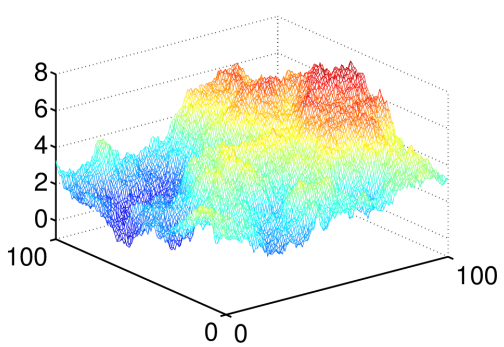

(a) Undeformed surface $\left(A^{*}=0\right)$

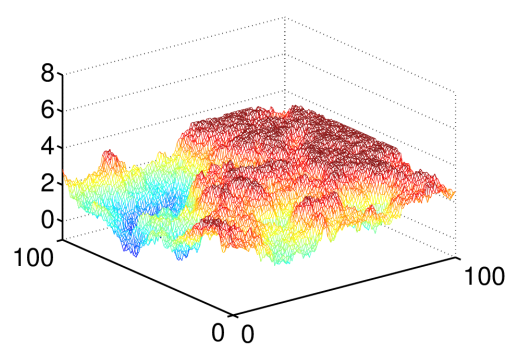

(b) Deformed surface $\left(A^{*} \sim 0.1\right)$

Figure 8: Original undeformed surface and its deformed shape corresponding to $A^{*} \sim 0.1$.

$L=100$ and $m=7$ in Fig $8(\mathrm{a})$ and its deformed shape corresponding to a real contact area fraction $A^{*} \sim 0.1$ in Fig $8(\mathrm{~b})$,

The spatial distribution of the real contact area and the amount of the free volume $v_{i, j}$ at each grid point can also be visualized. Here the total free volume is $V=\sum_{i, j} v_{i, j}$, where $i, j$ are indices running over all the boundary elements. Due to roughness, the asperities, which are the maxima of the $3 \mathrm{D}$ surface, come into contact at isolated points and then progressively merge together by forming wider contact regions with zero free volume. Other regions of the surface present free volumes $v_{i, j}$ whose size depends on the amplitude of the valleys. A contour plot in Fig 9 corresponding to the deformed configuration in Fig 8(b) displays the areas with different values of $v_{i, j}$. The dark red color denotes the contours having the highest values of $v_{i, j}$, whereas the deepest blue represents the contour for $v_{i, j}=0$. The morphological properties of the surface valleys, usually neglected in contact problems where the real contact area is the primary quantity of interest, are indeed relevant for the spatial distribution of the free volume. Existing standards suggest to use the indices $S b i$ and $S v i$ to quantify the free volume properties of surfaces. In particular, the valley fluid retention index, $S v i$, computed from the bearing area curve as the volume comprised between the undeformed surface and the plane leaving only $20 \%$ of the heights below it, and divided by the product $\sigma A_{n}$, is certainly a useful indicator to distinguish between surfaces with very large or small valleys. However, these roughness parameters computed from the original undeformed geometry do not account for the effect of asperity deformations occurring during contact.

A deeper insight into the morphological properties of the spatial distribution of the free volume can be made by examining the contour levels corresponding to different volume thresholds, $v_{t h}$, as shown in Fig 10 . In these contours, the black area denotes domains $\mathbb{D}$ where $v_{i, j} \leq v_{t h}$. Therefore, the dark islands for the limit case of $v_{t h}=0$ correspond to the real contact area domain. Selecting $v_{t h}$ larger than the maximum value of the volume of the deepest valley, $v_{t h}=$ $\max \left(v_{i, j}\right)$, the picture becomes entirely black since all the elementary areas of the grid have $v_{i, j} \leq v_{t h}$. This second limit situation corresponds to the Euclidean domain of the nominal contact area. 


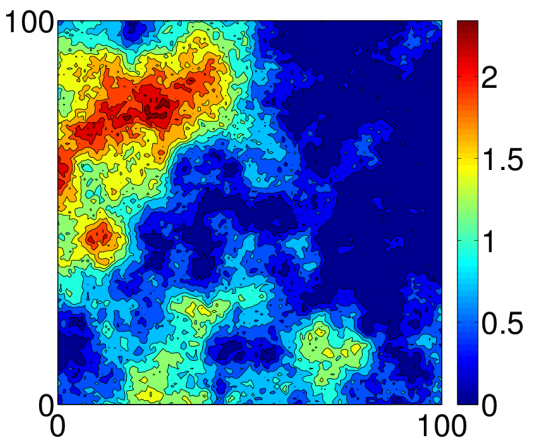

Figure 9: Contour plot of the free volumes $v_{i, j}$ related to Fig $8(\mathrm{~b})$ Red denotes deep valleys with larger free volumes not in contact, blue denotes asperities in contact with zero free volume remaining (see the online version for colours).

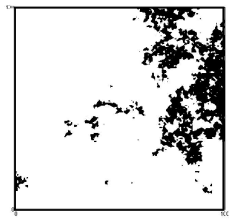

(a) $v_{\mathrm{th}}=0.098$

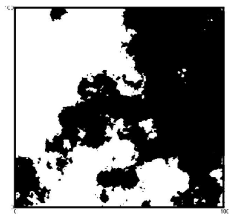

(d) $\mathrm{v}_{\mathrm{th}}=0.781$

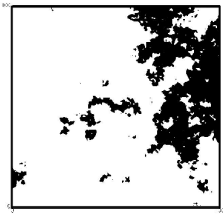

(b) $v_{\text {th }}=0.195$

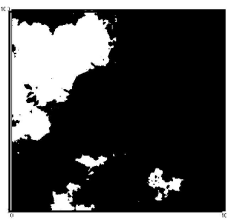

(e) $\mathrm{V}_{\mathrm{th}}=1.562$

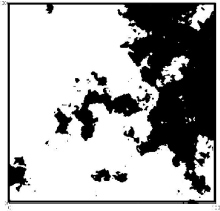

(c) $v_{\mathrm{th}}=0.391$

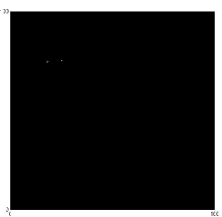

(f) $V_{\text {th }}=3.125$

Figure 10: Free volume domains (black areas) $\mathbb{D}\left(v_{i, j} \leq v_{t h}\right)$ corresponding to Fig 9 for different free volume thresholds $v_{t h}$.

It has to be remarked that the contour plots in Fig 10 , corresponding to the same contact pressure and contact area, dynamically change during contact. At first contact, the real contact area $A^{*}$ is zero and the free volume $V^{*}$ is maximum. By increasing the contact pressure, the domain of the real contact area increases until it reaches $A^{*}=1$ and full contact takes place. Conversely, the free volume domain progressively shrinks down to zero in the same limit. It has to be pointed out that the observed scaling of the real contact area is in agreement with former numerical investigations, see [3, 7.

For each contour plot in Fig 10 , the topological properties of the free volume domains $\mathbb{D}\left(v_{i, j} \leq v_{t h}\right)$ can be investigated according to the box counting method. For $A^{*}=0$ (undeformed rough surface), the free volume domains are expected to be self-affine as a consequence of the self-affinity of the parent surface [46]. For a value $0<A^{*} \leq 1$, on the other hand, the topological properties of the 


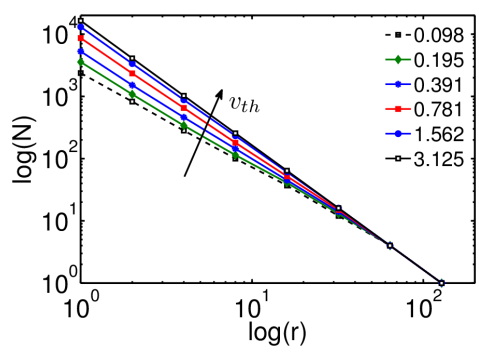

(a) Box-counting plot

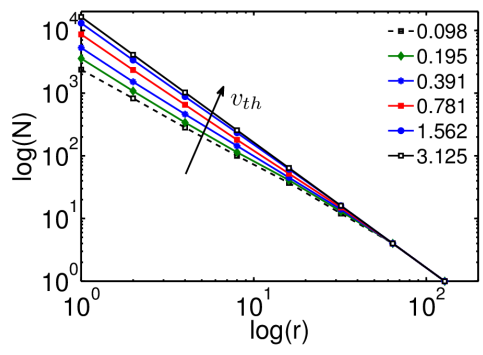

(b) Local fractal dimension

Figure 11: Fractal characterization of the free volume domains $\mathbb{D}\left(v_{i, j} \leq v_{t h}\right)$ shown in Fig 10 Fig 8(b) shows how the local fractal dimension $\mathcal{D}$ varies as a function of $r$ and $v_{t h}$.

free volume domains have to be correlated with those of the deformed surface whose heights have been modified by elastic deformation.

For each box of lateral size $r$, the number $N$ of boxes containing at least one black grid point are counted. This operation is repeated by varying $r$ from 1 up to $2^{m}$ lateral size divisions, with a geometric progression of 2 . The cumulative number $N(r)$ is plotted vs. $r$ in a bi-logarithmic diagram and the local fractal dimension $\mathcal{D}$ of the volume domain can be finally obtained by differentiating $\log (N)$ w.r.t. $\log (r)$.

By performing this analysis for the domains $\mathbb{D}$ in Fig 10 , we obtain the diagram in Fig.11(a). The curves have a trend close to a straight line in this bi-logarithmic plot, which suggests a power-law scaling typical of fractals.

The local fractal dimension is shown in Fig 11(b) and it is found to be dependent on $r$. In the limit case corresponding to $v_{t h}=0$, the fractal dimension of the corresponding free volume domain is equal to that of the real contact area, which is less than 2 due to the lacunarity of the contact domain. According to the results in [18, the fractal dimension of the contact area is an increasing function of the applied pressure but it is a decreasing function of $D$. In the other limit scenario of $v_{t h}=\max \left(v_{i, j}\right)$, the fractal dimension is equal to 2, i.e., it is equal to that of an Euclidean smooth surface. These limit values represent the bounds to the fractal dimension of the free volume contours $\mathbb{D}\left(v_{i, j} \leq v_{t h}\right)$ by varying $v_{t h}$.

\section{Theoretical considerations}

Apart from the direct post-processing of the numerical results, a theoretical formula for the computation of $V^{*}$ could be proposed by following the principle inspiring the bearing area curve [47, which is also the base for the definition of the valley fluid retention index Svi [44, 45]. According to this reasoning, the free volume for a given indentation $\Delta$ (related to the elevation $h$ of the contacting plane from the average plane of the surface heights determined in the undeformed configuration) can be estimated via the integral of the vertical 
gap $(h-z)$ times the distribution density function $\Phi(z)$ of the surface heights, multiplied by the real area not in contact:

$$
V^{*}=\frac{A_{n}-A_{r}}{\sigma A_{n}} \int_{-\infty}^{h}(h-z) \Phi(z) \mathrm{d} z=\frac{1-A^{*}}{\sigma} \int_{-\infty}^{h}(h-z) \Phi(z) \mathrm{d} z,
$$

where the integration limits span over the whole range of heights having a positive gap, i.e., from $-\infty$ up to $h$.

It is remarkable to note that, although this approach provides a decay of $V^{*}$ by increasing $A^{*}$, this would be simply linear if the distribution $\Phi(z)$ is assumed to be a Gaussian distribution, as often put forward in the literature (see [48] for a review of micromechanical contact theories making this assumptions). This is not exactly the case observed in numerical experiments, see Fig 3(b). Moreover, the predictions of Eq.(4) are independent of $D$ and $m$, unless an additional relation $A^{*}(D, m)$ is invoked. Hence, according to Eq. (4), the dependency of $V^{*}$ on the fractality of surfaces and on their resolution would be an indirect consequence of the dependency of $A^{*}$ on $D$ and $m$, as also takes place in the model based on Persson's theory 37.

An alternative and more accurate path to evaluate the free volume is to exploit the fractal properties of the corresponding domains $\mathbb{D}$ analyzed for instance in Fig 11. This approach leads to a formula for the estimation of $V^{*}$ independent from Eq. (4). This has the advantage of being able to explain the dependencies of $V^{*}$ on the fractal dimension and on the surface resolution solely from the statistical distribution of the free volume, without invoking the scaling properties of other contact predictions like the real contact area.

The main consequence of the plot in Fig.11(a) is that the cumulative number of boxes with a volume $v_{i, j}<v_{t h}$ has a power-law scaling with respect to the box size $r$ :

$$
N(r)=(R / r)^{\mathcal{D}}
$$

where $R$ is a free parameter to be determined from data. Equation (5) can be recast in terms of a lateral size $l$ and of the sampling interval $\delta$, with again $l$ as a free parameter:

$$
N(\delta)=(l / \delta)^{\mathcal{D}}
$$

Here, $\mathcal{D}$ should be considered as dependent on $r$ and $v_{t h}$, according to the trends shown in Fig, 11(b).

For a given surface resolution, the total free volume $V^{*}$ can be computed according to the following integral:

$$
V^{*}=\frac{1}{\sigma A_{n}} \int_{0}^{v_{t h, \max }} n \mathrm{~d} v_{t h}
$$

where $n$ represents the number of boxes with a free volume comprised in the range $v_{t h}<v_{i, j}<v_{t h}+\mathrm{d} v_{t h}$. 
A closed-form expression to $n$ appears to be difficult to be derived, since this is a nonlinear function of $v_{t h}$. However, it is possible to proceed with a numerical integration of Eq.(7) by suitably partioning the limit of integration $v_{t h, \max }$ in $K$ intervals $\left(v_{t h, k}, v_{t h, k+1}\right)$, where $k=1, \ldots, K$, and replacing the integration by a discrete sum. The number of boxes $n$ can be approximated by the finite difference between $N\left(v_{t h, k+1}\right)$ and $N\left(v_{t h, k}\right)$ :

$$
n_{k} \cong(l / \delta)^{\mathcal{D}_{k+1}}-(l / \delta)^{\mathcal{D}_{k}},
$$

where $\mathcal{D}_{k}$ and $\mathcal{D}_{k+1}$ denote the fractal dimensions of the free volume domains corresponding to $v_{t h, k}$ and $v_{t h, k+1}$, respectively.

The free volume of the surface comprised in the range $v_{t h, k}<v_{i, j}<v_{t h, k+1}$ is therefore:

$$
\Delta V_{k}=n_{k} \Delta v_{t h},
$$

where $\Delta v_{t h}=v_{t h, k+1}-v_{t h, k}$. The total volume $V^{*}$ can be finally determined as

$$
V^{*}=\frac{1}{\sigma A_{n}} \sum_{k=1}^{K} \Delta V_{k}
$$

Equation (7) can be used to interpret the numerical trends observed in Section 3.1 via the dependency of the fuction $n\left(v_{i, j}\right)$ on $D$ and $\delta$. However, instead of examining $n\left(v_{i, j}\right)$, it is more elegant and general to investigate the probability density function $f(v)$ that can be deduced from the numerically determined histograms $n\left(v_{i, j}\right)$. The values of the probability density function $f\left(v_{i, j}\right)$ are simply determined from $n\left(v_{i, j}\right)$ as $n\left(v_{i, j}\right) / n_{\text {tot }}$ and imposing the condition $\int_{0}^{v_{t h, \max }} f(v) \mathrm{d} v=1$.

As an example, let us focus on the contact configurations corresponding to a maximum indentation of the half-plane such that its final position coincides with the average plane of the originally undeformed rough surface. The probability density function $f(v)$ is determined from BEM results corresponding to the fractal surfaces with different $D$ and $m$ whose contact response has been shown in Section 3.1.

Investigating the effect of the surface fractal dimension on the free volume, we observe that $f(v)$ is a decreasing function of $v$ with an approximately linear decay, see Fig.11(a). By decreasing $D, v_{t h \text { max }}$ is reduced and the probability density function becomes steeper and steeper. Interestingly, the point $f(v=1)$ is almost independent of $D$ and acts as a fulcrum about which the function $f$ rotates. Clearly, this effect has a double implication on the computation of $V^{*}$ according to Eq. (7): the integration interval defined by $v_{t h \text {, max }}$ is reduced by decreasing $D$ and the probability density function values increase for $v \rightarrow 0$. As a consequence, the value of the free volume $V^{*}$ at the maximum contact area (or at the maximum pressure) is diminishing by reducing $D$, thus explaining the trends highlighted in Fig 6 


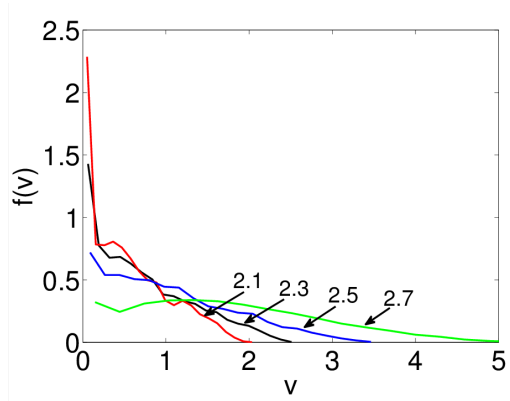

(a) The effect of $D(m=7)$

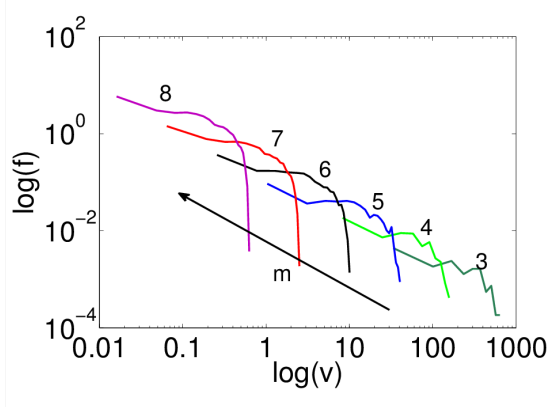

(b) The effect of $m(D=2.3)$

Figure 12: The effect of the surface fractal dimension $D$ and of the surface resolution related to the generation parameter $m$ on the probability distribution function $f(v)$ of the free volumes $v$.

As far as the effect of the surface resolution is concerned, we have to examine the plot of the probability distribution functions for surfaces having $D=2.3$ and different $m$ ranging from 3 to 8 , see Fig $12(\mathrm{~b})$. The function $f(v)$ is again a decreasing function of $v$ with an approximately linear decay as in Fig 12(a) However, changing the parameter $m$ leads to a significant shift in the probability density function values and in $v_{t h, \max }$, with variations of up to 4 orders of magnitude. Therefore, a bilogarithmic scale has been adopted in Fig 12(b) for visualization purposes. By increasing $m$, the increased value of $f$ for small values of $v$ is counterbalanced by the much smaller value of $v_{t h \text { max }}$. The net result stemming from the integration (7) is a decay in the total free volume $V^{*}$ by increasing $m$, which provides the explanation to the trends observed in Fig.7.

An explanation to the shift of the curves in Fig 12(b) by varying $m$ can be provided based on the theoretical findings in [49. By increasing $m$, we have an increase of the number of asperities and a decrease of the mean volume at the same time. If we denote by $\bar{f}$ the mean of $f$ and by $\bar{v}$ the mean of the free volume, one must have $\bar{f} \bar{v}=c$, where $c$ is a constant. Then $\log \bar{f}=-\log (\bar{v})+\log (c)$, which provides the slope -1 of the line connecting the average values $(\bar{v}, \bar{f})$ at different resolutions in Fig 12(b). Concerning the amount of the horizontal shift of the curves by varying $m$, one can assume that $\bar{v} \propto\left(L / 2^{m}\right)^{D}$. Taking the $\operatorname{logarithm}$ of this scaling law, we obtain $\log (\bar{v})=-D m \log (2)+\log c^{\prime}$, where $c^{\prime}$ is a constant. Hence, for $D=2.3$, we expect $\log (\bar{v})=-0.7 m+\log \left(c^{\prime}\right)$. A translation of $1 \mathrm{~m}$ unity gives a translation of 0.7 units in the horizontal coordinate, which is close to the actual shift of $\bar{v}$ from numerical data, see for instance $\log (8)-\log (2)=0.6$ by passing from $m=6$ to $m=7$.

\section{Conclusion}

In the present study, the dependency of the free volume between fractal rough surfaces in contact as a function of the real contact area and of the contact 
pressure has been studied by using the boundary element method. Two main aspects related to the surface morphological properties have been investigated, namely the effect of the surface fractal dimension $D$ and the effect of the surface resolution, which is related to the surface generation parameter $m$ of the random midpoint displacement algorithm.

Examining the relation between the free volume and the real contact area, we found a nonlinear decay. The free volume is diminished in case of small surface fractal dimensions (small $D$ ), or in case of very refined surfaces (high $m$ ). Concerning the relation between the free volume and the contact pressure, a nonlinear decay has been observed. While an increase in the surface fractal dimension provides a significant increase in $V^{*}$ for a given value of $p^{*}$, a surface refinement leads to a convergence towards a single curve. This is a notable behavior which does not take place for other contact relations, like for $A^{*}$ vs. $p^{*}$.

A detailed morphological analysis of the contour plot of the free volume has also been conducted. It has been found that the free volumes have a complex spatial distribution over the nominal cross-section area. The contour domains $\mathbb{D}$ corresponding to different free volume thresholds $v_{t h}$ present a local dimension $\mathcal{D}$ dependent on the scale of observation, bounded from below by the fractal dimension of the real contact area and from above by 2, i.e., by the Euclidean dimension of a flat surface.

Finally, the fractal properties of the free volume domain have been exploited in order to derive a formula for the computation of the free volume. Based solely on the probability distribution function of the free volumes, it allows a straightforward interpretation of the surface fractal dimension and surface resolution dependencies observed in the numerical BEM results.

Further theoretical and numerical research in this field is deemed to be important, especially as far as the rigorous study of the fluid-structure interaction is concerned, for instance in the case of a fluid squeezed among the contacting surfaces. This topic can be very important in mechanical engineering for a detailed modelling of lubrication by taking into account roughness, an issue important for wear. In fact, the development of an appropriate elastomer roughness in radial lip seals has been found to be dependent on the surface roughness of the shaft 32 . Optimized roughness properties and texturing can increase the performance of oil seals, significantly reducing wear 50, and controlling the direction of leakage flow through the sealing interface. In general, the control of the seal film thickness, which is directly related to the free volume between the rough surfaces in contact, can be beneficial in reducing the forces acting on the mechanical system and increasing the sealing capabilities [51. In case of random roughness, an accurate computation of the free volume between surfaces in contact and of the statistical parameters related to the valley distributions can be efficiently achieved based on the computational method herein proposed, fully accounting for the elastic deformation of the interface. Hence, the proposed method can be applied to textured surfaces as well, since there are no restrictions on the form of the height field given in input.

Other technological applications relevant for the present study regard inter- 
faces in composites for energy applications, like photovoltaic modules. In those cases, the nonuniform thickness of polymer seals induced by roughness can be dangerous for the long-term reliability of these systems exposed to environmental conditions. Humidity percolation and the evolution of chemical degradation of the electric contact between Silicon and the deposited silver conductors is in fact strongly correlated to imperfect sealing and voids [52]. The characterization of how leakage mechanisms take place in those systems is of paramount importance and tailoring of surface roughness may lead to a new generation of devices with improved lifetime.

\section{Acknowledgements}

MP would like to thank the Université Paris EST for supporting his visiting full professorship in the Laboratoire de Modélisation et Simulation Multi Echelle during May 2014. The research leading to these results has received funding from the European Research Council under the European Union's Seventh Framework Programme (FP/2007-2013) / ERC Grant Agreement n. 306622 (ERC Starting Grant "Multi-field and multi-scale Computational Approach to Design and Durability of PhotoVoltaic Modules" - CA2PVM). The authors would like to thank the anonymous Reviewer for having brought to their attention the scaling law underlying Fig.12(b).

\section{References}

[1] J.A. Greenwood, J.B.P. Williamson, Contact of nominally flat surfaces. Proc. Roy. Soc. Lond. Ser. A 295 (1966) 300-319.

[2] A. Majumdar, B. Bhushan, Role of fractal geometry in roughness characterization and contact mechanics of surfaces, ASME J. Tribol. 112 (1990) 205-216.

[3] M. Borri-Brunetto, A. Carpinteri, B. Chiaia, Scaling phenomena due to fractal contact in concrete and rock fractures. Int. J. Fract. 95 (1999) 221238.

[4] B.N.J. Persson, Theory of rubber friction and contact mechanics, J. Chem. Phys. 115 (2001) 3840-3861.

[5] G. Carbone, F. Bottiglione, Asperity contact theories: Do they predict linearity between contact area and load? J. Mech. Phys. Sol. 56 (2008) $2555-2572$.

[6] M. Paggi, M. Ciavarella, The coefficient of proportionality $\kappa$ between real contact area and load, with new asperity models, Wear 268 (2010) 10201029.

[7] V.A. Yastrebov, G. Anciaux, J.F. Molinari, From infinitesimal to full contact between rough surfaces: evolution of the contact area. International Journal of Solids and Structures 52 (2015) 83-102. 
[8] M.G. Cooper, B.B. Mikic, M.M. Yovanovich, Thermal contact conductance. Int. J. Heat Mass Transfer 12 (1968) 279-300.

[9] M. Ciavarella, V. Delfine, G. Demelio, A "re-vitalized" Greenwood \& Williamson model of elastic contact between fractal surfaces, J. Mech. Phys. Solids 54 (2006) 2569-2591

[10] M. Ciavarella, S. Dibello, G. Demelio, Conductance of rough random profiles. Int. J. Solids Struct. 45 (2008) 879-893.

[11] M. Ciavarella, J.A. Greenwood, M. Paggi, Inclusion of "interaction" in the Greenwood and Williamson contact theory. Wear 265 (2008) 729-734.

[12] R. Pohrt, V.L. Popov, Normal contact stiffness of elastic solids with fractal rough surfaces. Phys. Rev. Lett. 108 (2012) 104301.

[13] R. Pohrt, V.L. Popov, Contact stiffness of randomly rough surfaces. Sci. Rep. 3 (2013) doi:10.1038/srep03293.

[14] J.R. Barber, Bounds on the electrical resistance between contacting elastic rough bodies. Proc. Roy. Soc. Lond. Ser. A 459 (2003) 53-66.

[15] M. Paggi, J.R. Barber, Contact conductance of rough surfaces composed of modified RMD patches. Int. J. Heat Mass Trans. 54 (2011) 4664-4672.

[16] L. Kogut, K. Komvopoulos, Electric contact resistance of elastic-plastic rough surfaces. Journal of Applied Mechanics, 94 (2003) 3153-3162.

[17] Z.-Q. Gong, K. Komvopoulos, Thermomechanical analysis of semi-infinite solid in sliding contact with a fractal surface. Journal of Tribology, 127 (2005) 331-342.

[18] M. Borri-Brunetto, B. Chiaia, M. Ciavarella, Incipient sliding of rough surfaces in contact: a multiscale numerical analysis. Comp- Meth. Appl. Mech. Eng. 190 (2001) 6053-6073.

[19] M. Paggi, R.Pohrt, V.L. Popov, Partial-slip frictional response of rough surfaces, Sci. Rep. (2014) 4, 5178.

[20] G. Carbone, L. Mangialardi, B.N.J. Persson, Adhesion between a thin elastic plate and a hard randomly rough substrate. Phys. Rev. B 70 (2004), 125407.

[21] G. Carbone, M. Scaraggi, U. Tartaglino, Adhesive contact of rough surfaces: comparison between numerical calculations and analytical theories. Eur. Phys. J. E 30 (2009) 65-74.

[22] G. Carbone, L. Mangialardi, Hydrophobic properties of a wavy rough substrate. Eur. Phys. J. E 16 (2005) 67-76. 
[23] M Nosonovsky, B Bhushan, Roughness optimization for biomimetic superhydrophobic surfaces. Microsystem Tech. 11 (2005) 535-549.

[24] J. Yang, K. Komvopolulos, A mechanics approach to static friction of elastic-plastic fractal surfaces. Journal of Tribology, 127 (2005) 315-324.

[25] W.R. Chang, I. Etsion, D.B. Bogy, An elastic-plastic model for the contact of rough surfaces, Journal of Tribology, 109 (1987) 257-263.

[26] Z. Song, K. Komvopoulos, Adhesive contact of an elastic semi-definite solid with a rigid rough surface: strength of adhesion and contact instabilities. International Journal of Solids and Structures, 51 (2014) 1197-1207.

[27] S.M.T. Chan, C.P. Neu, K. Komvopulos, A.H. Reddi, The role of lubricant entrapment at biological interfaces: reduction of friction and adhesion in articular cartilage. Journal of Biomechanics, 44 (2011) 2015-2020.

[28] B. Berkowitz, Characterizing flow and transport in fractured geological media: A review. Advances in Water Resources 25 (2002) 861-884.

[29] A. Tarabay, Advanced computation models for the evolution of fracture networks in shale during hydraulic fracturing. Proc. 1st Int. Symp. Energy Challenges and Mechanics, July 8-10, 2014, Aberdeen, Scotland, UK.

[30] B. Han, Measurements of true leak rates of MEMS packages. Sensors 12 (2012) 3082-3104.

[31] C.K. Green, Development of a Leakage Model for Solid Oxide Fuel Cells Compressive Seals. PhD Thesis, Georgia Institute of Technology, 2007.

[32] W.J. Leachman, H. Li, T.J. Flynn, L.S. Stephens, C.A. Trinkle, Statistical analysis of wear of biplanar deterministically-arrayed surfaces for load bearing applications. Wear 311 (2014) 137-148.

[33] B. Yu, P. Cheng, A fractal permeability model for bi-dispersed porous media. Int. J. of Heat Mass Transfer 45 (2002) 2983-2993.

[34] Q. Zheng, J. Xu, B. Yang, B. Yu, A fractal model for gaseous leak rates through contact surfaces under non-isothermal condition. Appl. Therm. Engng. 52 (2013) 54-61.

[35] B. Lorenz, B.N.J. Persson, Leak rate of seals: Comparison of theory with experiment. European Phys. Lett. 86 (2009) 44006.

[36] B. Lorenz, B.N.J. Persson, On the dependence of the leak rate of seals on the skewness of the surface height probability distribution. European Phys. Lett. 90 (2010) 38002.

[37] F. Bottiglione, G. Carbone, L. Mangialardi, G. Mantriota, Leakage mechanism in flat seals. J. Appl. Phys. 106 (2009) 104902. 
[38] C. Vallet, D. Lasseux, P. Sainsot, H. Zahouani, Real versus synthesized fractal surfaces: Contact mechanics and transport properties. Trib. Int. 42 (2009) 250-259.

[39] H.O. Peitgen, D. Saupe, The Science of Fractal Images, Springer-Verlag, New York, 1988.

[40] G. Zavarise, M. Borri-Brunetto, M. Paggi, On the resolution dependence of micromechanical contact models, Wear 262 (2007) 42-54.

[41] K.L. Johnson, Contact Mechanics, Cambridge University Press, Cambridge, UK, 1985.

[42] J.R. Barber, Elasticity, Springer, Dordrecht, 3rd edn., 2010.

[43] A. Bemporad, M. Paggi, Optimization algorithms for the solution of the frictionless normal contact between rough surfaces, submitted.

[44] K.J. Stout, P.J. Sullivan, W.P. Dong, E. Mainsah, N. Luo, T. Mathia, H. Zahouani, The development of methods for the characterization of roughness on three dimensions. Publication no. EUR 15178 EN of the Commission of the European Communities, Luxembourg (1994).

[45] Surface Texture (Surface Roughness, Waviness and Lay) ANSI/ASME B46.1, American Society of Mechanical Engineers, NY, New York 10017, 1995.

[46] M. Bigerelle, A. Iost, Statistical artefacts in the determination of the fractal dimension by the slit island method. Engineering Fracture Mechanics, 71 (2004) 1081-1105.

[47] E.J. Abbott, F.A. Firestone, Specifying surface quality: a method based on accurate measurement and comparison. Mechanical Engineering, 55 (1933) $569-572$.

[48] G. Zavarise, M. Borri-Brunetto, M. Paggi, On the reliability of microscopical contact models, Wear, 257 (2004) 229-245.

[49] M. Bigerelle, J.-M. Nianga, D. Najjar, A. Iost, C. Hubert, K.J. Kubiak, Roughness signature of tribological contact calculated by a new method of peaks curvature radius estimation on fractal surfaces. Tribology International 65 (2013) 235-247.

[50] M. Gawlinski, Optimal surface roughness of the shafts co-operating with oil lip seals. BHR Group Conference Series Publication, Bury Sr. Edmunds (2000) 309-320, Professional Engineering Publishing, 1998.

[51] R.B. Siripuram, L.S. Stephens. Effect of deterministic asperity geometry on hydrodynamic lubrication. ASME Journal of Tribology 126 (2004) 527-534. 
[52] M. Van Iseghem, A. Plotton, J.-F. Penneau, N. Chatagnon, D. Binesti, K. Radouane, P.-G. Therond. Failure modes after damp heat tests on PV modules. 27th EU PVSEC European PV Solar Energy Conference and Exhibition, 4BV.3.47. 